\title{
An Evaluation Method of the Photovoltaic Power Prediction Quality
}

\author{
Mao Yang $(1)$ and Xin Huang \\ School of Electrical Engineering, Northeast Electric Power University, Jilin 132012, China \\ Correspondence should be addressed to Mao Yang; yangmao820@163.com
}

Received 22 August 2017; Revised 29 December 2017; Accepted 13 February 2018; Published 15 March 2018

Academic Editor: Emilio Turco

Copyright ( 2018 Mao Yang and Xin Huang. This is an open access article distributed under the Creative Commons Attribution License, which permits unrestricted use, distribution, and reproduction in any medium, provided the original work is properly cited.

Photovoltaic (PV) output power has regularity, volatility, and randomness. First of all, this paper carried on a metrological analysis to PV system data. Then, this paper analyzed the relationship between PV historical data, PV power forecasting model, and forecast error. By spectrum analysis of PV power, the PV power is decomposed into periodic components, low frequency residual components, and high frequency residual components. Making a specific analysis of these three components determines the minimum modeling error value, which reflects the unpredictable part of the PV power. Determining the minimum modeling error for PV forecasting not only objectively evaluates the quality of the PV forecasting model but also can determine the prediction accuracy standard according to different PV power generation targets. The examples given in this paper illustrate the effectiveness of the method.

\section{Introduction}

Renewable energy power is an important solution to global warming. Solar energy generated from PV systems is one of the fastest and the most promising growing renewable energy types [1]. PV power has a large randomness and volatility due to the light intensity, humidity, battery temperature, and so forth [1-3]. This randomness and volatility of PV output cause some adverse effects on the grid when it is connected in a large scale. Therefore, accurate PV power prediction is of great significance to the safe and economical operation of power system.

The prediction of PV power is to use a certain modeling method based on historical data [1,3-5]. In recent years, a large number of studies have focused on the method of PV power prediction. In [2], based on the analysis of the system structure, two-phase orthogonal currents were constructed, the DC component of the reactive current of the loads is acquired by the $i_{p}-i_{q}$ algorithm based on the instantaneous reactive power theory, the $\mathrm{DC}$ component of the active current is derived from the PI controller, and thus the gridconnected command current is obtained. The distance analysis method was employed in [5] to analyze the correlation between PV power generation and weather factors. In order to adapt to the weather mutation, the self-organizing feature map was used to identify the weather clustering from the cloud forecast information; the corresponding forecasting network was used for each weather class. In [6], a combined forecasting model was proposed based on the rough set. Three kinds of single prediction models were firstly established based on similarity date, support vector machine, and persistence forecasting method. Then, the weight was then assigned to the forecast produced by each prediction model through determining their attribute importance in rough set theory. In the literature [3], considering the influence of wind speed and light on the power flow of microgrids, the prediction of the combined probability distribution of microgrid trend can reduce the adverse effect of wind speed and mild randomness on microgrid operation. According to the prediction of wind power and PV power generation, the qualitative prediction of the trend of microgrid was carried out, and then the conditional joint probability distribution and the unconditional joint probability distribution of the microgrid trend were predicted by combining the Markov chain.

The above methods only focus on finding effective forecasting methods and do not take into account the predictability and unpredictability of the PV power time series itself. In this paper, the regularity of the PV power is fully excavated, 
as well as its physical explanation, so as to achieve the greatest modeling accuracy. Firstly, the periodic characteristics of PV power are analyzed. Then, the PV power is decomposed by Fourier decomposition to extract the corresponding periodic component including daily cycle components, low frequency components, and high frequency components which are analyzed and explained physically. Finally, the minimum modeling error is determined from the high frequency components. In order to verify the effectiveness of the method, the minimum error of PV power is analyzed under different forecast horizons and different locations. The minimum modeling error determined is then compared with the standard deviation of the prediction error obtained from the three PV power prediction modeling methods, that is, continuous method, artificial neural networks, and generalized regression audit network of PV power generation combined forecast, respectively. The prediction of PV power is similar to the load forecasting, and the research on the evaluation of the load regularity has existed. The necessity of the evaluation of the load regularity has been expounded in [10], and a method of load regularity evaluation was put forward based on statistical analysis. The minimum modeling error of the PV power can be obtained by the method of PV power regularity. When the error is compared with the results of each prediction model, the quality of each model can be evaluated objectively. Furthermore, the result obtained may provide a reference for the relevant departments to develop the forecasting error standard for a specific PV power plant.

This paper is organized as follows. Section 2 carries on a metrological analysis to PV system data. Section 3 analyzes the relationship between the prediction error and the regularity of the PV power. Section 4 demonstrates an evaluation method of PV power regularity. Section 5 analyzes the modeling error. Section 6 verifies the effectiveness of the proposed method. Section 7 offers the conclusions of this study.

\section{The Metrological Analysis to PV System Data}

When sunlight shines on the surface of a solar cell, its semiconductor interface converts light energy into electrical energy due to the PV effect. Solar cell output power by the solar radiation intensity, temperature, humidity and wind speed, and other factors, the equivalent circuit shown in Figure 1 . In the figure, $I_{\mathrm{ph}}$ is the current generated by the photovoltaic cell, which is strongly related to the light-receiving area and the illumination condition of the PV cell; $I_{d}$ is the equivalent diode current; $R_{\mathrm{sh}}$ is the bypass resistor, which has a great resistance value and can be neglected in the ideal circuit; $R_{s}$ is the series resistance; $R_{1}$ is the equivalent load resistance of PV cells; $I$ is the load current; $V$ is the load voltage.

In order to facilitate the analysis of PV cell characteristics and avoid complicated calculations, the practical equivalent of PV cells can be approximated to meet the required accuracy in practical engineering applications, and an equivalent model of PV cells can be established [11].

$$
\begin{aligned}
I_{1} & =I_{\mathrm{Ph}}\left(1-C_{1}\left(e^{(V-V D) / C_{2} V_{\mathrm{oc}}}-1\right)\right)+\mathrm{DI} \\
C_{1} & =\left(1-\frac{I_{m}}{I_{\mathrm{ph}}}\right) e^{V_{m} / C_{2} V_{\mathrm{oc}}}
\end{aligned}
$$

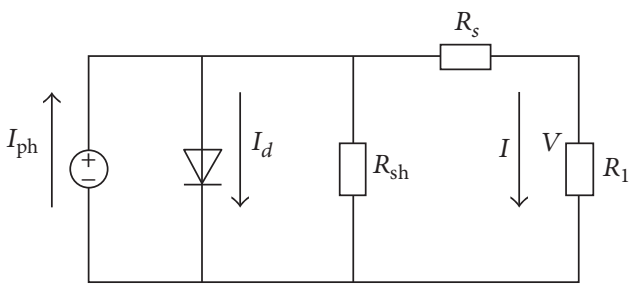

Figure 1: Equivalent circuit of PV cell.

$$
\begin{aligned}
C_{2} & =\frac{\left(V_{m} / V_{\mathrm{oc}}-1\right)}{\ln \left(1-I_{m} / I_{\mathrm{ph}}\right)} \\
D I & =\alpha \frac{G}{G_{\mathrm{ref}}}\left(t-t_{\mathrm{ref}}\right)+I_{\mathrm{ph}}\left(\frac{G}{G_{\mathrm{ref}}}-1\right) \\
D V & =-\beta\left(t-t_{\mathrm{ref}}\right)-R_{s} \mathrm{DI}
\end{aligned}
$$

$V_{\mathrm{oc}}$ is the open circuit voltage of PV cells; $I_{m}$ is the maximum power point current; $V_{m}$ is the maximum power point voltage; $G$ is the actual solar radiation intensity; $G_{\text {ref }}$ is the standard solar irradiation intensity, $G_{\text {ref }}=2500 \mathrm{~W} / \mathrm{m}^{2}$; $t$ is the actual temperature; $t_{\text {ref }}$ is the standard temperature; $\alpha$ is the standard current change under solar radiation intensity-temperature coefficient; $\beta$ is the voltage coefficient of variation at standard temperature.

The influence of solar radiation intensity on the output characteristics of PVcells was analyzed based on (1). The standard temperature $G$ is $1000 \mathrm{~W} / \mathrm{m}^{2}, 1500 \mathrm{~W} / \mathrm{m}^{2}$, $2000 \mathrm{~W} / \mathrm{m}^{2}$, and $2500 \mathrm{~W} / \mathrm{m}^{2}$. The IV characteristic and PV characteristic curve of a certain type of PV cell were obtained by using MATLAB/Simulink simulation software, as shown in Figure 2. As can be seen from Figure 2, changes in the intensity of solar radiation mainly affect the size of the shortcircuit current of the PV cell, but the influence on the open circuit voltage is not obvious. With the continuous increase of solar radiation intensity, the short-circuit current of PV cells is getting larger and larger, and the maximum output power of PV cells also increases.

As the PV power by a variety of complex factors, such as temperature, humidity, wind speed, and component status. Therefore, it is impossible to predict the PV power $100 \%$ accurately. How to determine the unpredictable degree of PV power sequence is the goal of this paper.

\section{Relationship between Prediction Error and Regularity of PV Power}

The PV power can be predicted due to its regularity. This rule can be represented by modeling PV power based on historical data within a training window of a specified length. The modeling error is

$$
\varepsilon_{I}(t)=P(t)-M(t) \quad t \in D^{-} .
$$

Set the PV power history data in the time domain to $D^{-}$and the predicted PV power data in the time domain to $D^{+}$. For 


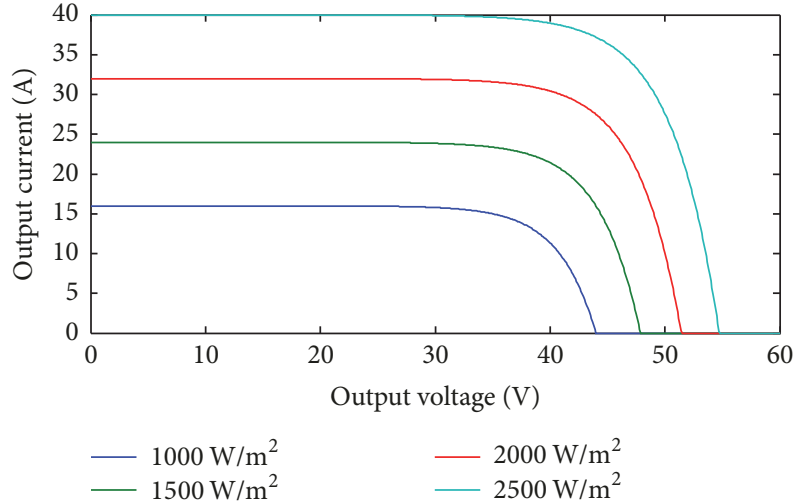

(a) $I-V$ characteristic curve

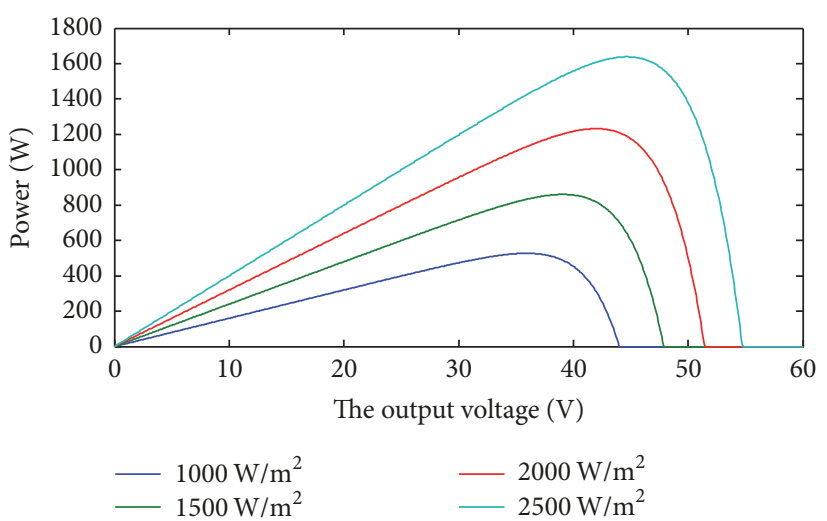

(b) $P-V$ characteristic curve

FIGURE 2: Effect of radiation intensity on PV output.

the PV power history data with the specified window width, if the PV power prediction model is obtained by a certain method, define $M(t)$ as the response of the model within historical time domain, define $P(t)$ as the history data of PV power.

The total error of the PV power prediction can be expressed as

$$
\varepsilon_{\Sigma}(t)=\varepsilon_{I}(t)+\varepsilon_{E}(t) \quad t \in D^{+}
$$

$\varepsilon_{I}(t)$ is the PV power error component and $\varepsilon_{E}(t)$ is extrapolation error. When the PV power of the second day is predicted by $M(t)$, if the PV power mode of the forecast day is the same as the PV power mode in $D^{-}$, the PV power error component with the same statistical characteristic as $\varepsilon_{I}(t)$ will continue into $D^{+}$with possibility of extrapolation error $\varepsilon_{E}(t)$.

This paper focuses on the analysis of the influence of modeling error on the prediction accuracy of PV power and its composition [12].

If the PV power curve for each day of the historical data (total $N_{d}$ days) for PV power prediction modeling is identical, then any day of the PV power curve is repeated $N_{d}$ times as the response $M(t)$ of the PV power model, where the PV power predictive modeling error $\varepsilon_{I} \equiv 0$. If the PV power of $\left(N_{d}+1\right)$ is predicted on this basis, there may be two cases: if the predicted PV power is exactly the same as the PV power curve in the modeling time domain, the extrapolation error $\varepsilon_{E}(t) \equiv 0$. The total prediction error $\varepsilon_{\Sigma}(t) \equiv 0$; if the predicted $\mathrm{PV}$ power is not exactly the same as the daily PV power curve in the modeling time domain, the deviation is $\Delta P(t)$ :

$$
\varepsilon_{\Sigma}(t)=0+\varepsilon_{E}(t)=\Delta P(t) \neq 0 \quad t \in D^{+} ;
$$

there is no modeling error at this time and the extrapolated error is the total error.

The special case of the above analysis is only to illustrate the relationship between the errors, and it can not be directly used for the actual PV power prediction error analysis. In fact, according to any one or more of the PV power modeling methods, it is always possible to decompose a set of PV sequences into

$$
P(t)=\sum_{i=1}^{k m} M_{i}(t)+\sum_{i=1}^{k u} U_{i}(t)=M(t)+U(t) \quad t \in D^{-},
$$

where $M_{i}(t)$ is the response of the regular PV power model, which will contribute to the prediction of the corresponding components of the future PV power; $U_{i}(t)$ is the PV power component without substantial contribution to the future prediction accuracy of the PV power; $\mathrm{km}$ and $\mathrm{ku}$ are the response and no substantive contribution to the number of components.

Comparing (2) and (4), the modeling error is

$$
\varepsilon_{I}(t)=U(t) \quad t \in D^{-} .
$$

From the above analysis, the size of the modeling error is related to the modeling method and the regularity of $P(t)$. If the method has been determined, the law of poor PV power corresponds to a larger modeling error; if the PV power has been determined, the better modeling method corresponds to a smaller modeling error.

In the practice of $\mathrm{PV}$ power prediction, the relative error of the prediction error is the percentage of the PV power value at the corresponding time. As an important index, the relative modeling error can be defined as follows:

$$
\varepsilon_{I}^{*}(t)=\frac{U(t)}{P(t)} \quad t \in D^{-} .
$$

The statistical characteristics of the relative modeling error is closely related to the prediction accuracy of PV power. For the actual PV power data, despite the effort to improve the PV power modeling method, it can not make the modeling error infinite to 0 . Because the $\mathrm{PV}$ power has a certain degree of randomness, in the process of improving the PV power prediction method, the error of modeling generally has a nonzero lower limit, which mainly reflects the inherent nonregularity of the PV power. For PV power data, it is important to estimate the relative error and determine the upper limit of the prediction accuracy. 


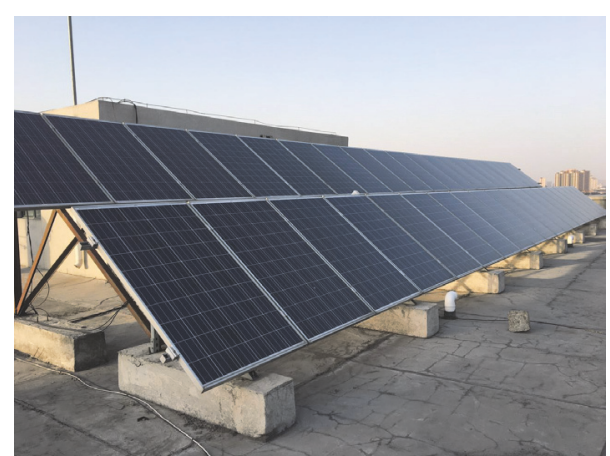

FIGURE 3: Distributed PV panels.

\section{Evaluation Method of PV Regularity}

4.1. Spectrum Analysis of PV Power Sequences. PV power sequence significantly satisfies the Dirichlet condition [13]. So the PV power signal can be developed into a Fourier series, which can be expressed as a series of different frequencies of sine or complex exponential signal sum.

The finite element Fourier decomposition of the PV power time series in a given modeling time domain is decomposed as follows [14]:

$$
\begin{array}{r}
X_{t}=a_{0}+\sum_{i=1}^{N / 2-1}\left[a_{i} \cos \left(\frac{2 \pi i t}{N}\right)+b_{i} \sin \left(\frac{2 \pi i t}{N}\right)\right] \\
+a_{N / 2} \cos (\pi t)=a_{0}+\sum_{i=1}^{N / 2} R_{i} \cos \left(\omega_{i} t+\Phi_{i}\right) \\
N=0,1,2, \ldots
\end{array}
$$

The cosine items are orthogonal to each other. Using this method, the variation of the PV power can be decomposed into the angular frequency component of $2 \pi / N, 4 \pi / N, \ldots, \pi$ which can be reconstructed by appropriate combination.

For the intuitive and effective representation of the signal contained in the components of the energy, it can make the spectrum [15]. The energy of the harmonic as the ordinate is defined as follows:

$$
W(t)=\frac{2\left|\operatorname{FFT}\left(X_{t}\right)\right|}{N},
$$

where FFT is the first-order fast Fourier transform function and the transformation result is complex. The spectrum can reflect the periodic characteristics of the PV power history data, and the high energy corresponds to the high periodic frequency.

Most of data used in this paper come from the National Key Laboratory of Northeast China Electric Power University, Jilin Province, China. The sampling interval is 15 minutes. Details of the PV test platform are shown in Table 1. Figure 3 is the school distributed PV cell device. Figure 4 is the PV power data acquisition platform.

The first part of Figure 5 shows the 14-day PV power raw signal output, which includes different weather types; the
TABLE 1: PV power plant information.

\begin{tabular}{lc}
\hline Item & Data \\
\hline Longitude & 126.5098 \\
Latitude & 43.8294 \\
Altitude & $80 \mathrm{~m}$ \\
Azimuth & $0^{\circ}$ \\
Tilt & $37^{\circ}$ \\
Single capacity & $25 \mathrm{~W}$ \\
Sampling interval & $15 \mathrm{~min}$ \\
Sampling time & $2015-2017$ \\
System storage & $64 \mathrm{G}$ \\
Mounting disposition & Flat roof \\
Field type & Fixed tilted plane \\
Installed capacity & $10 \mathrm{kWp}$ \\
Material & Polycrystalline silicon \\
PV module & $\mathrm{JKM} 245 \mathrm{P}$ \\
The type of sensor & $\mathrm{ZZ-S}-\mathrm{COMB}-\mathrm{B}$ \\
Accuracy of humidity & $3 \% \mathrm{RH}\left(25^{\circ} \mathrm{C}\right)$ \\
Accuracy of temperature & $0.5^{\circ} \mathrm{C}\left(25^{\circ} \mathrm{C}\right)$ \\
Accuracy of radioactivity & $7 \% \mathrm{~W} / \mathrm{m}^{2}\left(25^{\circ} \mathrm{C}\right)$ \\
\hline
\end{tabular}

second part shows the spectrum of the signal; the last part shows details of the spectrum.

The data are collected from 6 am to $5 \mathrm{pm}$. Through the observation of the spectrum, from which the maximum energy of the three corresponding to the frequency of analysis, the extraction results are as shown in Table 2.

The first high energy corresponding frequency is converted to a period of about 12 hours. The time of the collected data per day is 12 hours. Through extensive analysis of different PV power data, the daily cycle characteristics of all PV power data are obvious because the daily periodicity of the light determines the daily cycle of the PV power. This also confirms the daily cycle of PV power output from a mathematical point of view.

4.2. Frequency Domain Decomposition of PV Power Sequences. The power data are decomposed by spectral analysis. The respective components in equation (8) are recombined into the following four components:

$$
P(t)=a_{0}+D(t)+L(t)+H(t),
$$

where $a_{0}$ is the constant component and its value is

$$
a_{0}=\frac{1}{T} \int_{t_{0}}^{t_{0}+T} x(t) d t ;
$$

$D(t), L(t)$, and $H(t)$ are composed of the sum of several harmonic components in (7). $D(t)$ is a periodic component with a period of 12 hours; $L(t)$ is a component whose period is greater than $12 \mathrm{~h} ; H(t)$ is a component whose period is $15 \mathrm{~min}-12 \mathrm{~h}$.

The finite element Fourier decomposition of the PV power time series in a given modeling time domain is decomposed as shown in Figure 6 [16]. 
TABLE 2: High energy frequency extraction.

\begin{tabular}{lccc}
\hline Item & First high energy & Second high energy & Third high energy \\
\hline Frequency $[\mathrm{Hz}]$ & $2.3180 \times 10^{-5}$ & $4.967 \times 10^{-6}$ & $1.8213 \times 10^{-5}$ \\
Cycle $[\mathrm{h}]$ & 12 & 56 & 15.25 \\
\hline
\end{tabular}

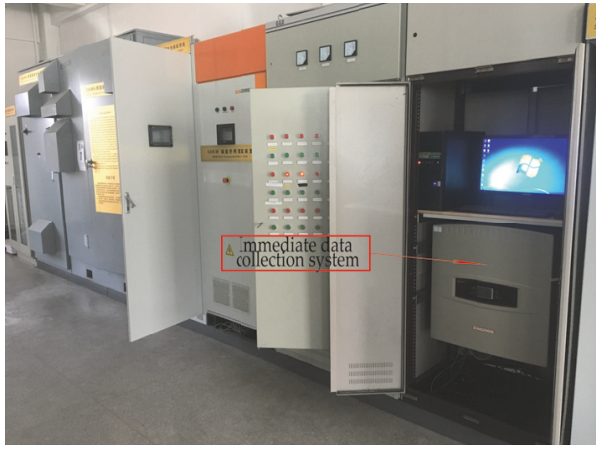

(a) Immediate data collection system

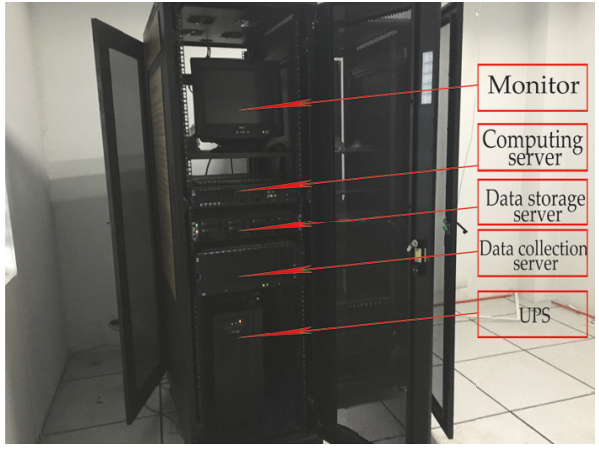

(b) PV power data storage system

FIGURE 4: PV power data acquisition platform.

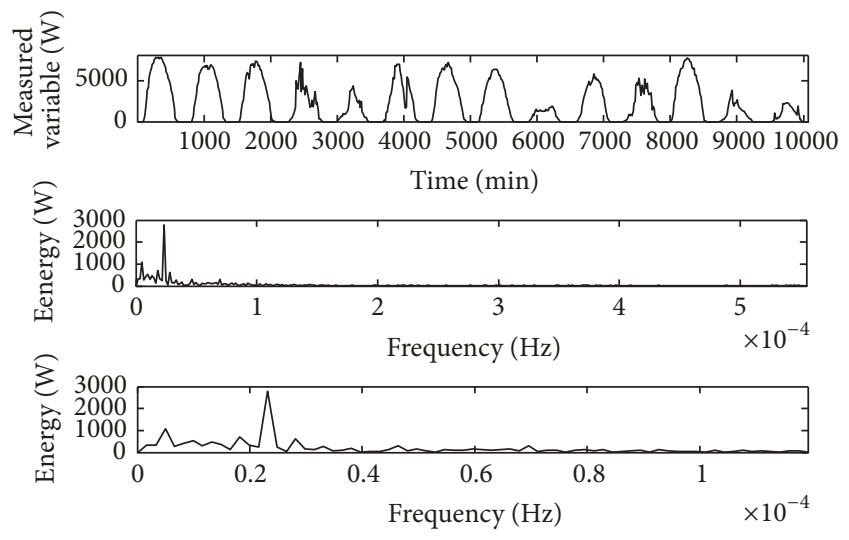

FIGURE 5: PV power curve and spectrum from 2015/01/01 to 2015/01/14.

Figure 4 shows the results of a group of PV power data (totaling $14 \mathrm{~d}$ ). The period of $D(t)$ is 96 time intervals, which is a component of $12 \mathrm{~h}$ in the PV power cycle. The daily period component is added to the constant component $a_{0}$, and $a_{0}+D(t)$ can be used as the periodic component of the $\mathrm{PV}$ power. After removing $a_{0}$ and $D(t)$ in $P(t)$, the remaining components can be regarded as the sum of the low frequency residual component $L(t)$ and the sum of the high frequency residual components and $H(t)$. The low frequency residual component reflects the influence of meteorological factors such as cloud block, temperature, and other factors on the PV power. $H(t)$ mainly reflects the randomness of PV power changes.

It can be seen from the observation that the amplitude of the low frequency residual fraction is relatively large, and the amplitude of the low frequency residual component can not directly reflect the influence of the variable-related factors such as weather on the PV output.
4.3. Analysis of Low Frequency Residual Components. The low frequency residual component contains some related factors to affect the PV power, and the cycle of low frequency is greater than $12 \mathrm{~h}$. If the relevant factors $x_{1}(t), x_{2}(t), \ldots, x_{k x}(t)$ are known to the impact of PV power, we can build the model:

$$
L_{m}(t)=f\left(x_{1}(t), x_{2}(t), \ldots, x_{k t}(t)\right) ;
$$

it can get the remaining decomposition of the low frequency:

$$
L(t)=L_{m}(t)+L_{u}(t),
$$

where $L_{m}(t)$ is a modeling part; $L_{u}(t)$ is a nonmodeling part.

Practice has proved that the low frequency residual component of the cycle is greater than $12 \mathrm{~h}$; low frequency residual component modeling will usually have a certain improvement in PV power prediction.

4.4. Analysis of High Frequency Residual Components. Because the cycle of high frequency is less than $12 \mathrm{~h}$, it is difficult to predict it. According to the time series analysis point of view, we can use the time series analysis method to model the high frequency residual components. By analyzing the variation characteristics of PV power in different periods at different time periods, the high frequency residual component autocorrelation function and the partial correlation function are as shown in Figure 7.

It can be seen from Figure 7, a second-order autoregressive model can be used [17].

$$
\begin{array}{r}
H^{\prime}(t)=\phi_{1} H(t-\Delta t)+\phi_{2} H(t-2 \Delta t)+e_{t} \\
\Delta t=15 \mathrm{~min},
\end{array}
$$

where $e_{t}$ is white noise; $\phi_{1}$ and $\phi_{2}$ are regression coefficients; $H^{\prime}(t)$ is the high frequency residual component predicted by AR modeling. If the high frequency residual component 


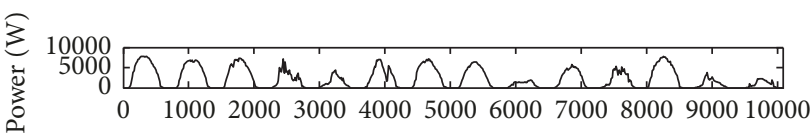

(a) Actual output time from 2015/01/01 to 2015/01/14 [min]

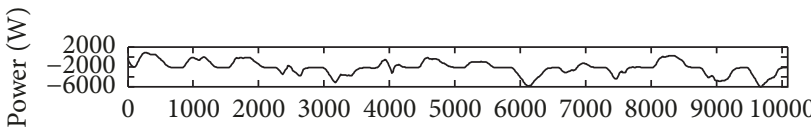

(c) Low frequency residual component time from 2015/01/01 to 2015/01/14 [min]

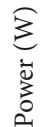

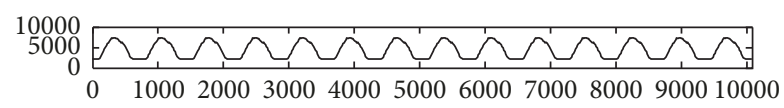

(b) Day cycle component time from 2015/01/01 to 2015/01/14 [min]

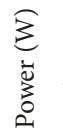

2000 2000

(d) High frequency residual component time from 2015/01/01 to 2015/01/14 [min]

FIGURE 6: Decomposition results of PV power in a region from 2015/01/01 to 2015/01/14.
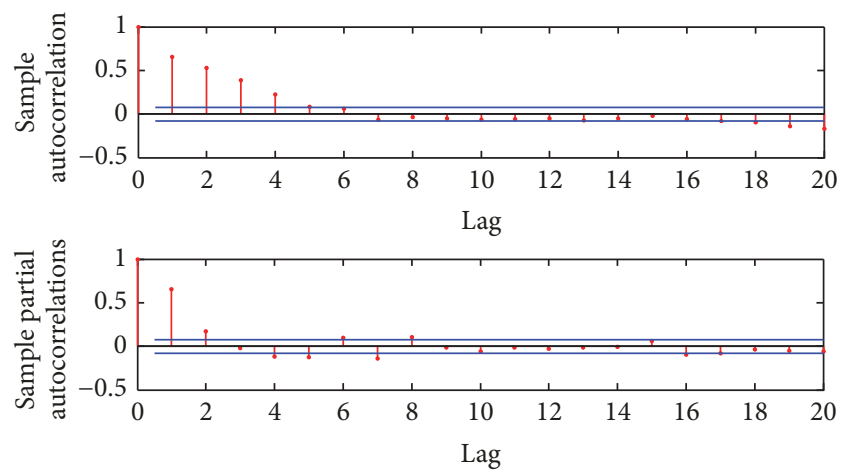

FIGURE 7: Canonical autocorrelation function and partial correlation function of high frequency residual component.

is used as a nonmodeling component $U(t)$, the error in the prediction of the PV power prediction will depend primarily on the standard deviation of $H(t)$; if the time series model in (11) can be used to predict the high frequency residual components of the PV power, $U(t)=e_{t}$, the modeling error will depend largely on the standard deviation of $e_{t}$, and $\sigma_{H}>$ $\sigma_{e}$.

The time-series modeling of the high frequency residual components in Figure 4 is given by the following equation:

$$
\begin{array}{r}
H^{\prime}(t)=2 H(t-\Delta t)-1.0814 H(t-2 \Delta t)-0.3432 \\
\Delta t=15 \mathrm{~min} .
\end{array}
$$

The modeling results are shown in Figure 8.

In fact, since the PV power prediction is a multistep prediction, it is required to perform extrapolated prediction of multistep (at least 48 steps) without supplementing the new sample observations. When the high frequency residual component is predicted by the time series model of the high frequency residual component, the number of steps is predicted. According to the literature [8], the step prediction error is estimated:

$$
E[\operatorname{er}(l)]^{2}=\left(1+\Psi_{1}^{2}+\cdots+\Psi_{l-1}^{2}\right) \sigma_{e}^{2},
$$

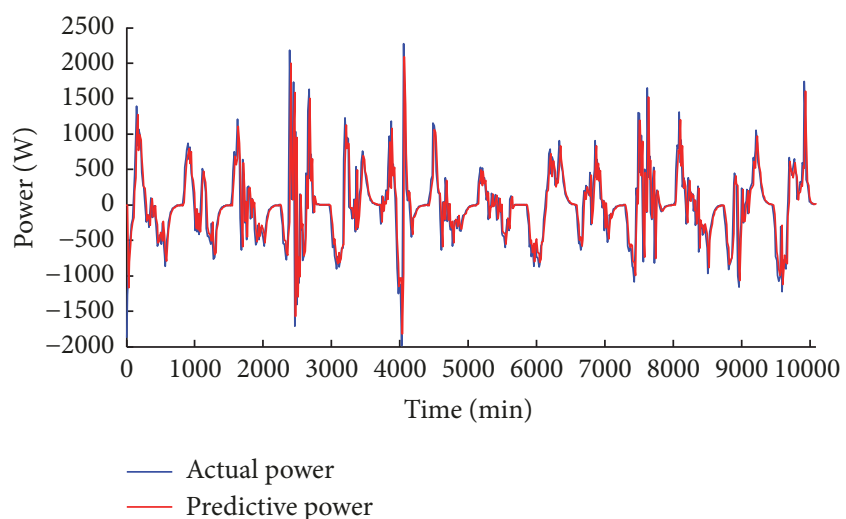

FIGURE 8: Comparison of actual power and AR prediction power of high frequency residual component.

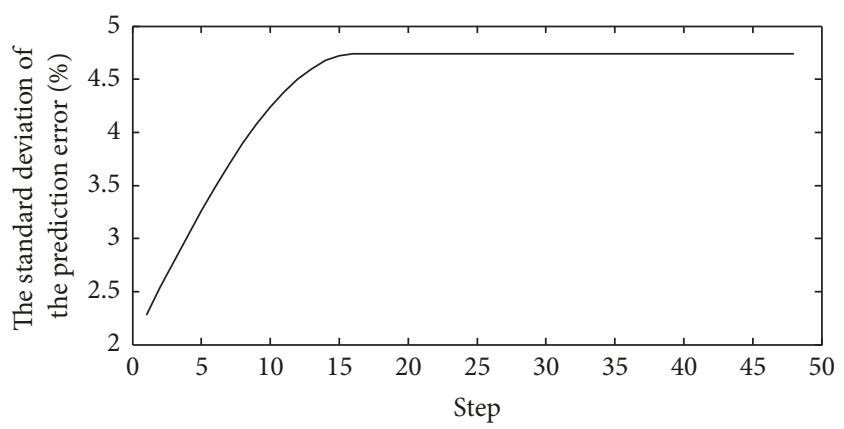

FIGURE 9: Standard deviation curve of multistep prediction error.

where $E$ is expected value; $\operatorname{er}(l)$ is predictive error of step $l ; \sigma_{e}$ is standard deviation of white noise; $\Psi_{i}(i=0,1, \ldots, l-1)$ is coefficient which can be calculated from $\phi_{1}$ and $\phi_{2}$.

$$
\sigma_{\text {er }}(l)=\sqrt{E[\operatorname{er}(l)]^{2}} .
$$

$\sigma_{\mathrm{er}}(l)$ is the standard deviation of $\operatorname{er}(l)$.

Figure 9 shows the variation of the standard deviation of the multistep prediction error of a high frequency component of a PV power with the number of steps.

Figure 9 shows that the standard deviation of the prediction error rises rapidly from $\sigma_{e}=2.28 \%$ to the standard 
TABLE 3: The standard deviation of the relative modeling error of the different PV power components with no real contribution to the prediction accuracy is defined.

\begin{tabular}{lcr}
\hline Item & $U(t)=H(t)$ & $U(t)=L(t)+H(t)$ \\
\hline Area A [\%] & 4.43 & 5.23 \\
Area B [\%] & 4.56 & 5.78 \\
Area C [\%] & 5.20 & 5.63 \\
\hline
\end{tabular}

deviation $\sigma_{H}=4.74 \%$ of the sequence high frequency residual component as the number of predicted steps increases. For the extrapolated 48-point PV power prediction, the $\mathrm{AR}(2)$ model can only improve the prediction accuracy of the first 16 points of the high frequency component, and the prediction accuracy of the 32 points is completely dependent on $\sigma_{H}$. Therefore, in the multistep prediction extrapolation conditions, $\sigma_{H}$ rather than $\sigma_{e}$ determines the high frequency residual component modeling error, so $H(t)$ is a component of $U(t)$. All standard deviations are obtained by dividing the standard deviation of the mathematical theory by the installed capacity.

The model methods used in this paper can be summarized in the flow chart shown in Figure 10. The minimum modeling error of the PV power can be determined using the method shown in the figure [11].

\section{Analysis of Modeling Error}

Define $\sigma_{I}$ and $\sigma_{I}^{*}$ as the standard deviation of modeling error and relative modeling error. The statistical characteristics of relative modeling error and modeling error are analyzed, which have a crucial effect on understanding the size of nonregular components in PV power.

If the PV power prediction method can only effectively predict the daily cycle component, then $M(t)=a_{0}+D(t)$, corresponding to $U(t)=L(t)+H(t)$; if the forecasting method can effectively predict the daily and weekly periods and low frequency residual components, then $M(t)=a_{0}+D(t)+L(t)$, corresponding to $U(t)=H(t)$.

The PV power data for 14 days in three different areas are divided into $U(t)$ by the above two methods. The sampling interval of the 14-day PV power data in the three regions is $15 \mathrm{~min}$, and the standard deviation of the relative modeling error is shown in Table 3. Area A is the National Key Laboratory of Northeast China Electric Power University; area B is Ashland PV Power Station in Oregon, USA; area C is Yellow River hydropower Golmud power station in China.

As can be seen from Table 3, the regularity of the PV power varies from region to region. When $U(t)=H(t)$ is taken, the lower limit of the prediction error of the PV power is corresponding, and the error evaluation can be made according to this lower limit.

Since the high frequency residual component is unpredictable in the actual prediction, the relative modeling error obtained by $U(t)=H(t)$ is taken as the minimum modeling error. The standard deviation of the minimum modeling error is $\sigma_{I(H)}^{*}$, which is the lower limit of the PV power prediction, and the upper limit of the PV power prediction accuracy can be determined, which is based on the minimum modeling error.
TABLE 4: Standard deviation of relative modeling error of regional B $\mathrm{PV}$ power in different periods.

\begin{tabular}{lcc}
\hline Item & $U(t)=H(t)$ & $U(t)=L(t)+H(t)$ \\
\hline Time 1 & 3.65 & 4.21 \\
Time 2 & 4.47 & 5.75 \\
Time 3 & 3.74 & 4.57 \\
Full time & & 4.84 \\
period & 3.95 & \\
\hline
\end{tabular}

Taking the PV power sequence $P_{A}(t)$ as an example, the probability distribution of the high frequency residual component is basically normal. Since $\sigma_{I(H)}^{*}=4.43 \%$, that is, the minimum modeling error is less than $4.43 \%$, the number of points in the total PV power is $68.3 \%$ and the minimum modeling error is less than $8.86 \%\left(2 \sigma_{I(H)}^{*}\right)$ of the points for the $95.4 \%$.

For the PV power sequence $P_{A}(t)$, when the error caused by the high frequency residual component is considered, if the specified qualified number of points is $97 \%$, the maximum prediction error of the qualified point is $\pm 9.61 \%$; if the prediction error is less than $3 \%$, qualified points will not exceed $50.17 \%$.

The regularity of PV power generation is different in time and space. Table 4 shows the standard deviation of the relative modeling error for the PV power of region B at different times. The data are based on a total of 42 days of output for the B region from March 1 to April 11, 2015, and the solar power data are modeled every fourteen days. It can be seen from Table 4 that the $\mathrm{B}$ region has the strongest regularity in the period 1 and the worst in period 2, which may be the change of the meteorological environment.

\section{PV Power Regularity Evaluation Results}

In order to show that the proposed method of PV power regularity evaluation is effective, the standard deviation of the actual prediction error of three different $\mathrm{PV}$ power prediction methods is compared with the standard deviation of the minimum modeling error in this paper. The historical data for the three regions are collected from October 1, 2015, to October 30, 2015, and the PV power for the day of October 31, 2015 , is predicted. The forecast results are shown in Table 5.

Table 6 shows the comparison between the standard deviation of the prediction error and the minimum modeling error for region $\mathrm{A}$ in different time periods. The time of data is from January 2015 to June 2015, and the data for each month is analyzed. Through the analysis of Tables 4 and 5, it can be seen that the standard deviation of the minimum 
TABLE 5: Comparison between the standard deviation of the actual prediction error and the standard deviation of the minimum modeling error.

\begin{tabular}{lcccc}
\hline \multirow{2}{*}{ Item } & \multirow{2}{*}{ The smallest modeling error [\%] } & \multicolumn{3}{c}{ Standard deviation of actual prediction error [\%] } \\
& & Method 1 [7] & Method 2 [8] & 5.22 \\
Area A & 3.55 & 4.42 & 6.13 & 5.85 \\
Area B & 4.72 & 5.24 & 6.25 & 5.01 \\
Area C & 5.19 & 5.58 & 6.34 \\
\hline
\end{tabular}

TABLE 6: Comparison of standard error and standard deviation of the standard deviation of the actual prediction error for each period of the A PV power.

\begin{tabular}{lcccc}
\hline Item & The smallest modeling error [\%] & \multicolumn{3}{c}{ Standard deviation of actual prediction error [\%] } \\
& & Method 1 [7] & Method 2 [8] & 5.156 \\
Method 3 [9] \\
\hline $2015-01$ & 4.123 & 4.454 & 4.645 & 4.825 \\
$2015-02$ & 3.930 & 4.359 & 4.958 & 5.698 \\
$2015-03$ & 4.516 & 4.989 & 5.605 & 4.684 \\
$2015-04$ & 4.587 & 5.648 & 4.788 & 5.869 \\
$2015-05$ & 4.432 & 4.685 & 5.687 & 6.698 \\
$2015-06$ & 5.123 & 5.658 & 5.140 & 5.685 \\
Average & 4.785 & 4.967 & & 5.743 \\
\hline
\end{tabular}

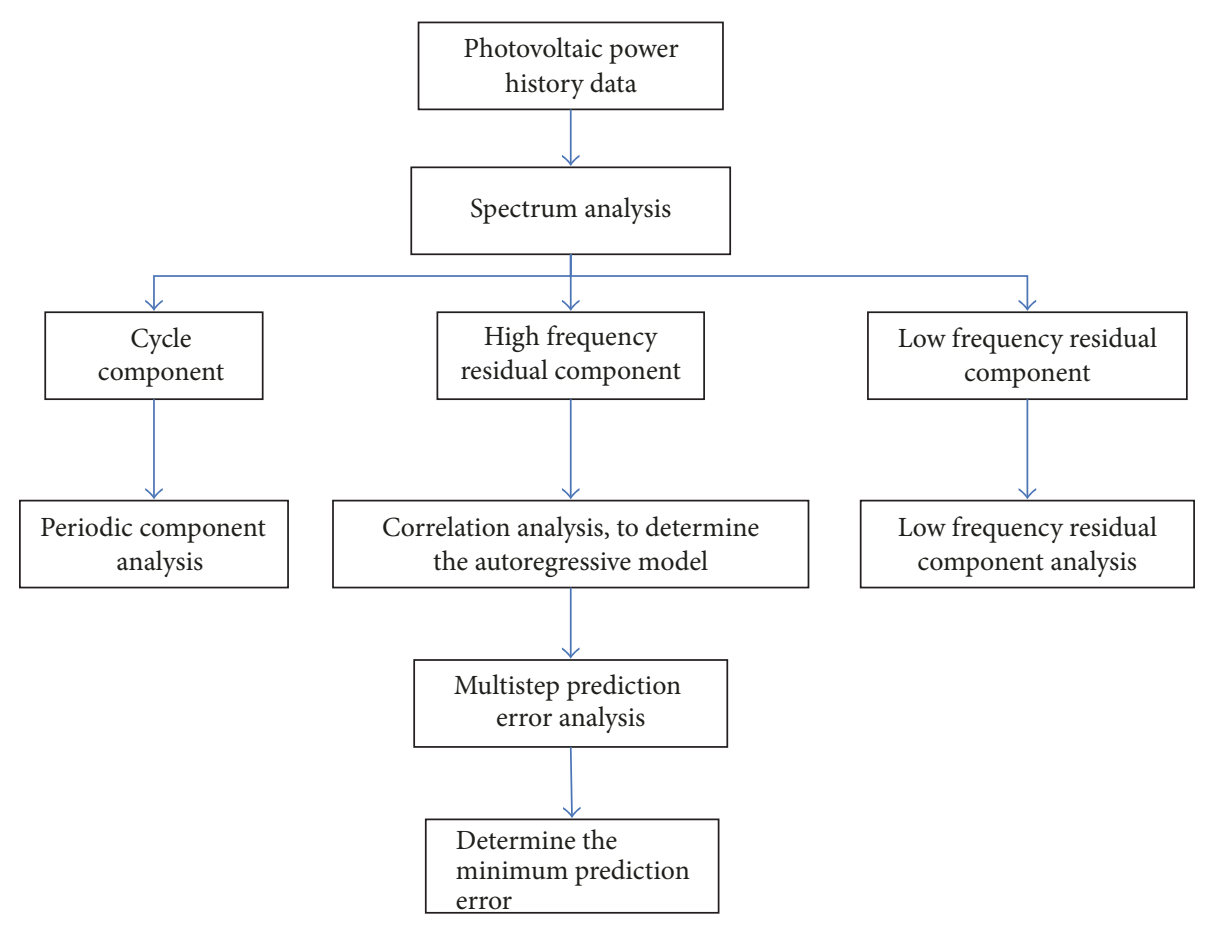

FIGURE 10: Model flow chart.

modeling error can reflect the difference of the regional and temporal characteristics of the PV power. At the same time, the minimum modeling error is positively correlated with the standard deviation of the actual prediction error. In Table 5, method 1 is preferred for region $A$ and region C; for region $B$, method 2 predicts the best results. Method 1 is the best in the prediction results in Table 6 . The standard deviation of each actual prediction error of all three methods is larger than the standard deviation of the minimum modeling error, which indicates that the estimation of the lower limit of the error is valid.

Method 1 is the continuous method, using the previous day's PV power output data as the next day PV power prediction data. Method 2 is wavelet decomposition and artificial networks, that is, with the ability of ANN to address nonlinear relationships, theoretical solar irradiance and meteorological variables are chosen as the input of the hybrid model based on WD and ANN. The output power of the PV plant is 
decomposed using WD to separate useful information from disturbances. The ANNs are used to build the models of the decomposed PV output power. Method 3 is combined with forecasting of PV Power Generation based on firefly algorithm-generalized regression auditing network. Firstly, to simplify model input dimensions, multiple linear factors influencing PV output are compressed and extracted with principal component analysis (PCA) method. Then the first principal component extracted from PCA combined with grey correlation degree is used to filter similar historical days. Next, the chosen days are, respectively, brought into two models, least square support vector machine (LS-SVN), and modified BP network (MBP), and the two predictions are repeated: the first is to forecast for similar day and then firefly algorithm for generalized regression neural network (FFAGRNN) is applied to train weight coefficients; the second is to ultimate forecast for test sets.

As can be seen from Table 6, the regularity of the specific time period in a particular region is different, the specific prediction method is not exactly the same for the regularity of the PV, and the minimum modeling error can well reflect the characteristics of $\mathrm{PV}$ power.

\section{Conclusion}

This paper presents A evaluation method of the PV power prediction quality. The variation of $\mathrm{PV}$ power varies with time and area, which is affected by light intensity, temperature, and other factors. There are inherent unpredictable factors in actual PV power generation, which are determined by the influence of some complicated factors and the characteristics of multistep prediction. Ignoring the inherent differences in $\mathrm{PV}$ power, it is impractical to unify the accuracy requirements of PV power forecasting. This supports a large number of PV power forecasting practices supported by the analytical methods in this paper. By using the concept of minimum modeling error presented here, the minimum modeling error can be determined by analyzing historical data of PV power, and the upper limit of the prediction accuracy of PV power can be estimated.

\section{Conflicts of Interest}

The authors declare that there are no conflicts of interest.

\section{Acknowledgments}

This work has been supported by Project 51307017 funded by the National Natural Science Foundation of China.

\section{References}

[1] P. Zhang, W. Li, S. Li, Y. Wang, and W. Xiao, "Reliability assessment of photovoltaic power systems: review of current status and future perspectives," Applied Energy, vol. 104, pp. 822-833, 2013.

[2] Y. Chen, A. Luo, and Z. Peng, "Robustpredictive control of PV grid connected generation and reactive power compensation,"
Transactions of China Electrotechnical Society, vol. 29, pp. 239246, 2013.

[3] M. Zhou, S. Ru, and J. Su, "Probabilistic power flow forecasting of microgrid based on joint probability distribution about wind and irradiance," Transactions of China Electrotechnical Society, vol. 29, pp. 55-63, 2014.

[4] Y. Gong, Z. Lu, and Y. Qiao, "Photovoltaic power prediction technology," Automation of Electric Power Systems, vol. 40, pp. 140-151, 2016.

[5] Y. W. Ma, H. L. Wu, and X. Y. Fang, "Optimal sizing of hybrid solar-wind distributed generation in an islanded micro-grid using improved bacterial foraging algorithm," Proceedings of the CSEE, vol. 31, no. 25, pp. 17-25, 2011.

[6] X. Yang, J. Ren, and Y. Xiao, "Integrated prediction model of photovoltaic output power based on Rough Set Theory," Chinese Power, vol. 49, pp. 133-138, 2016.

[7] M. Yang and B. Ji, "Wind power ultra short term prediction based on local first order weighted method," Journal of North China Electric Power University, vol. 35, pp. 06-10, 2015.

[8] H. Zhu, X. Li, Q. Sun, L. Nie, J. Yao, and G. Zhao, "A power prediction method for photovoltaic power plant based on wavelet decomposition and artificial neural networks," Energies, vol. 9, article 11, 2016.

[9] X. Wang, K. Huang, and Y. Zheng, "Prediction of photovoltaic power generation based on firefly algorithm and generalized regression audit network," Grid Tec, vol. 41, pp. 455-461, 2017.

[10] G. Mu, K. Hou, and Y. Yang, "Research on the evaluation method of load regularity," CSEE, vol. 10, pp. 96-101, 2001.

[11] M. Mao, S. Yu, and J. Su, "Versatile matlah simulation model for photovoltaic array with MPPT function," Journal of System Simulation, vol. 17, pp. 1248-1251, 2015.

[12] C. Wang, J. Lei, and A. Xu, "Ultra-short- term power output forecasting of distributed photovoltaic based on error classification," South. Power Sys Tec, vol. 9, pp. 41-46, 2015.

[13] Z. Fu, J. Wang, and G. Zhou, "Method of phase difference measurement based on the theory of initial phase harmonic wave," Elect Measu. Inst, vol. 52, pp. 76-80, 2015.

[14] M. Chatfield, The guide of time series analysis.1st, Yuhang Press, Beijing, China, 1996.

[15] B. Li, S. Chen, and S. Liang, "An output fluctuations of PV system energy storage capacity optimization method," Power Sys Prote. Control, vol. 42, pp. 45-50, 2014.

[16] M. Zhou, S. Ru, and J. Su, "Short term wind speed forecasting based on RVM and ARMA error correcting," Transactions of China Electrotechnical Society, vol. 27, pp. 187-193, 2012.

[17] B. George, Time series analysis: forecasting and control, Machinery Industry Press, Beijing, China, 4th edition, 2011. 


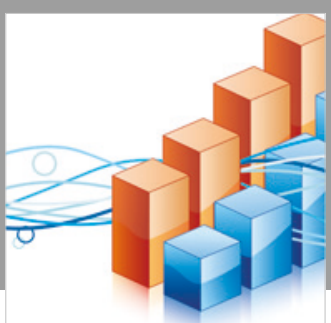

Advances in

Operations Research

\section{-n-m}
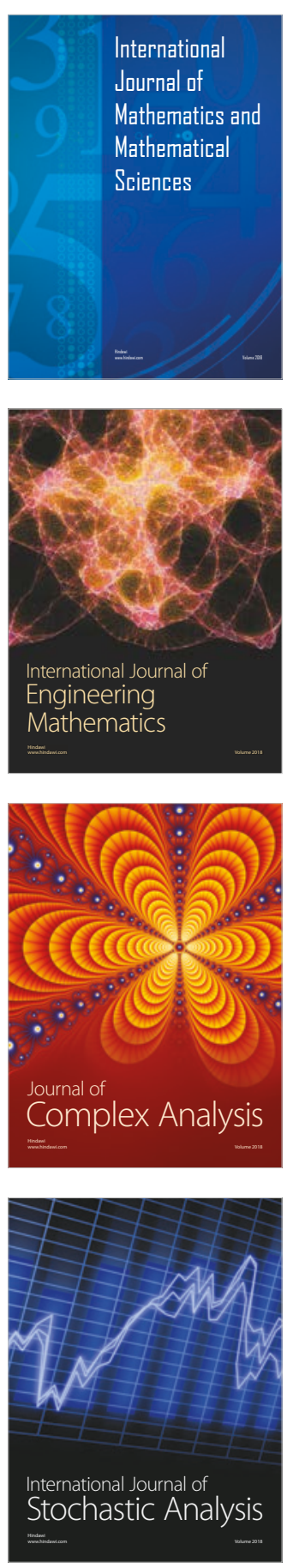
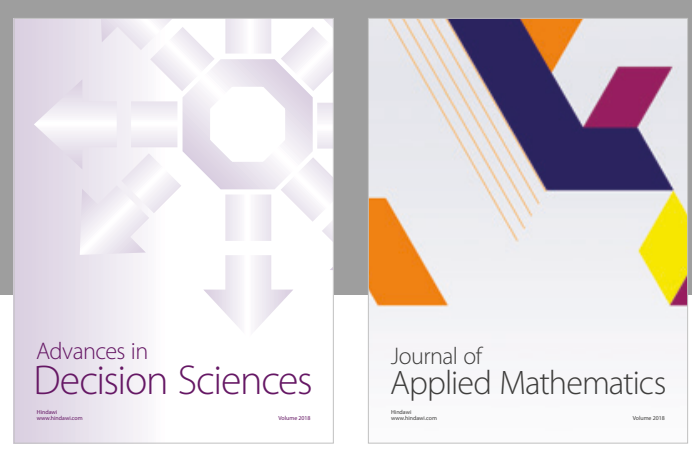

Journal of

Applied Mathematics
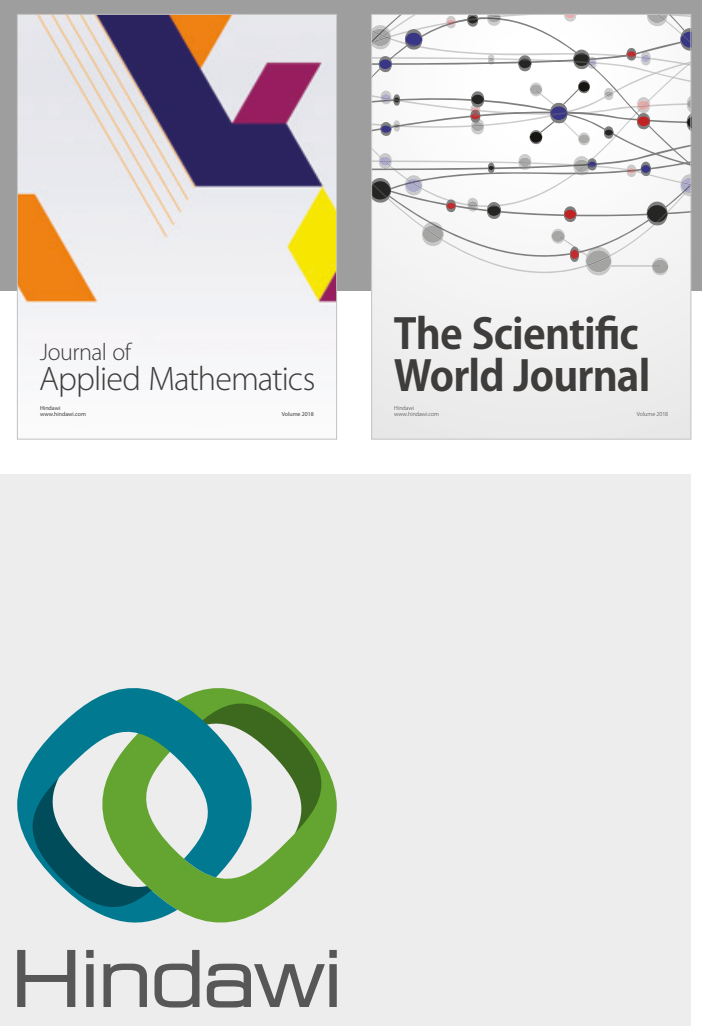

Submit your manuscripts at

www.hindawi.com

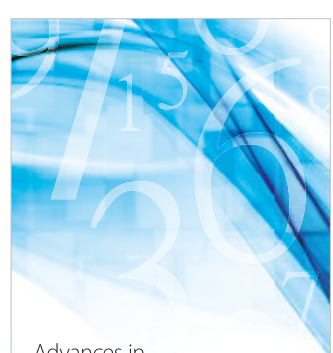

Advances in
Numerical Analysis
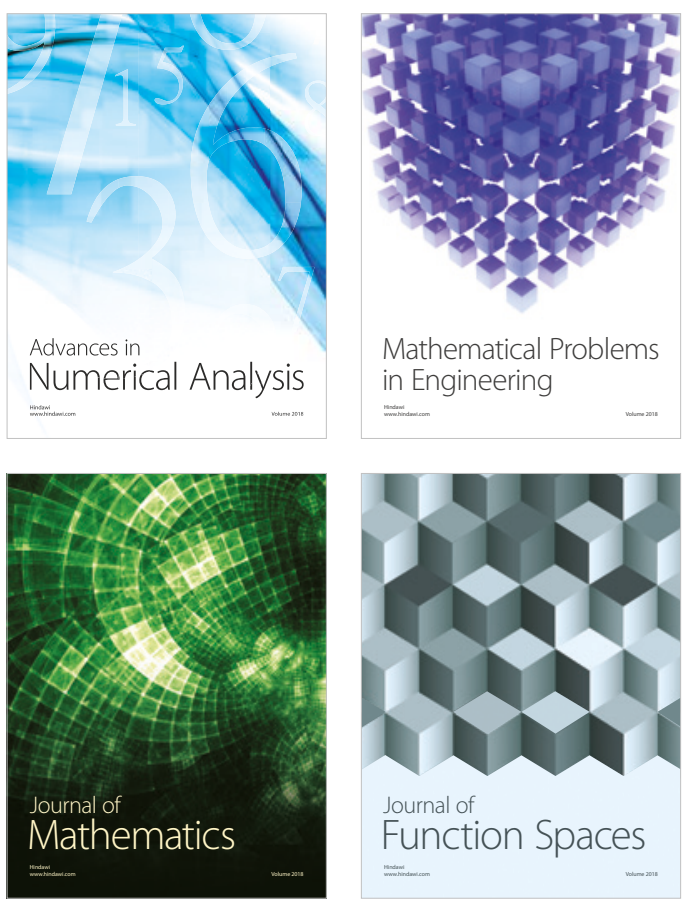

Mathematical Problems in Engineering

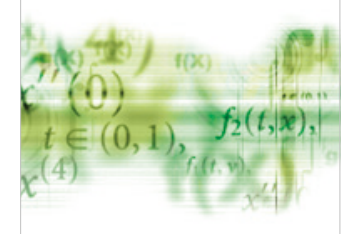

International Journal of

Differential Equations

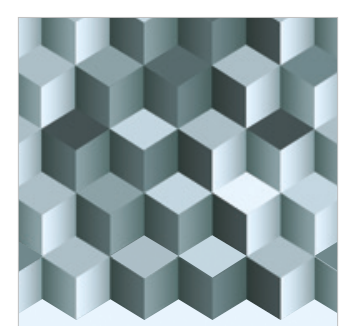

Journal of

Function Spaces

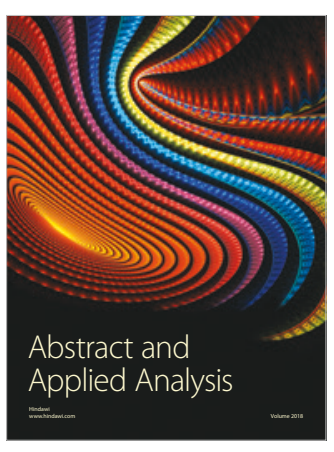

The Scientific

World Journal

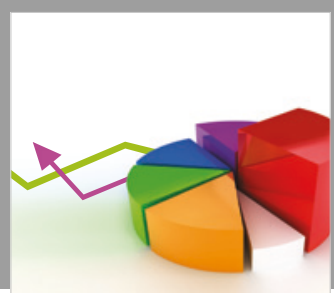

Journal of

Probability and Statistics
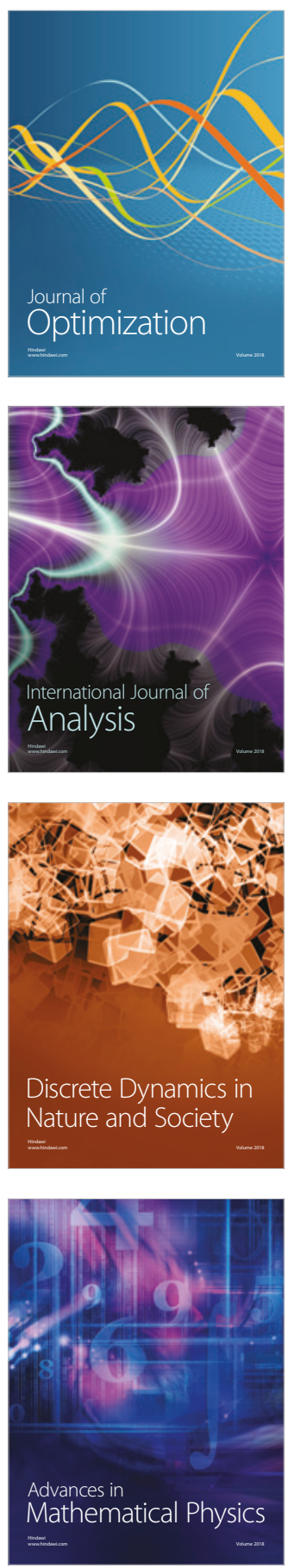